\title{
Circular RNAs in renal cell carcinoma: implications for tumorigenesis, diagnosis, and therapy
}

\author{
Ying Wang ${ }^{1}$, Yunjing Zhang ${ }^{1}$, Ping Wang ${ }^{2^{*}}$, Xianghui $\mathrm{Fu}^{3^{*}}$ and Weiqiang Lin ${ }^{1 *}$
}

\begin{abstract}
Renal cell carcinoma (RCC) is the most common malignant kidney tumor and has a high incidence rate. Circular RNAs (circRNAs) are noncoding RNAs with widespread distribution and diverse cellular functions. They are highly stable and have organ- and tissue-specific expression patterns. CircRNAs have essential functions as microRNA sponges, RNA-binding protein- and transcriptional regulators, and protein translation templates. Recent reports have shown that circRNAs are abnormally expressed in RCC and act as important regulators of RCC carcinogenesis and progression. Moreover, circRNAs have emerged as potential biomarkers for RCC diagnosis and prognosis and targets for developing new treatments. However, further studies are needed to better understand the functions of circRNAs in RCC. In this review, we summarize and discuss the recent research progress on RCC-associated circRNAs, with a focus on their potential for RCC diagnosis and targeted therapy.
\end{abstract}

Keywords: CircRNA, Renal cell carcinoma, Biomarker, Targeted therapy

\section{Background}

Renal cell carcinoma (RCC), the most common kidney neoplasm, originates in renal tubular epithelial cells and accounts for $85-90 \%$ of adult renal malignancies [1]. RCC is the sixth- and eight-most common cancer in males and females, and it is estimated that there will be 73,750 new cases of RCC in 2020 in the US [2]. RCC diagnoses have been increasing in recent years, mainly due to improvements in imaging techniques and ultrasonography $[3,4]$. Currently, surgical resection is the first-line treatment for RCC. However, local recurrence

\footnotetext{
*Correspondence: wpyyy@126.com; xfu@scu.edu.cn; wlin@zju.edu.cn 2Department of Urology, the First Affiliated Hospital, Zhejiang University School of Medicine, Hangzhou 310003, Zhejiang, China ${ }^{3}$ Division of Endocrinology and Metabolism, State Key Laboratory of Biotherapy and Cancer Center, West China Hospital, Sichuan University and Collaborative Innovation Center of Biotherapy, Chengdu 610041, Sichuan, China

'Kidney Disease Center, The Fourth Affiliated Hospital, Institute of Translational Medicine, Zhejiang University School of Medicine, Jinhua 322000, Zhejiang, China
}

or distant metastasis still occur in some patients even after radical nephrectomy. Moreover, the majority of RCCs are resistant to chemotherapy and radiotherapy once they recur or metastasize [5-7]. Although targeted therapies have significantly improved therapeutic outcomes for advanced RCC patients, their effectiveness is still limited. Cancer recurrence, metastasis, and resistance to therapy affect the recovery of patients with RCC [8-13]. Therefore, the early detection of postoperative micrometastases and recurrent lesions, and overcoming RCC drug resistance can improve patient prognosis.

RCC tumorigenesis is an extremely complex process that involves genetic mutations and the dysregulation of epigenetic pathways [14-20]. The tumor suppressor von Hippel-Lindau (VHL), one of the most frequently mutated genes in RCC, regulates the hypoxia pathway by controlling the activity of hypoxia-inducible factors (HIFs) [21]. The phosphoinositide 3-kinase (PI3K)/AKT/ mammalian target of rapamycin (mTOR) pathway is constitutively activated in RCC and plays a crucial role 
in regulating cell growth [22]. Concurrently, epigenetic changes in RCC such as DNA methylation [23], noncoding RNAs (ncRNAs), and histone modifications [24] have been extensively studied [25]. It has been demonstrated that numerous ncRNAs are dysregulated and involved in cancer initiation and progression. Thus, the potential application of targeting ncRNAs is currently an emerging area of clinical interest [26].

The discovery of ncRNAs has added to the understanding of how RCC develops and how it may be treated. It is generally recognized that most of the mammalian genome is transcribed into ncRNAs, including microRNAs (miRNAs) and long ncRNAs (lncRNAs). miRNAs, the best-studied class of ncRNAs, are involved in the pathogenesis of different cancers, including RCC. For example, miR-210 is upregulated in RCC and it regulates VHL, HIF-1, and HIF-2, which in turn promote RCC aggressiveness via multiple mechanisms [27]. Moreover, increased levels of miR-210 are directly correlated with poor patient outcomes, suggesting that it could be a potential biomarker for RCC diagnosis and prognosis [27]. The significance of miRNAs in RCC has been comprehensively summarized in a recent excellent review [28]. Accumulating evidence also suggests the involvement and potential importance of lncRNAs in gene regulation in human diseases, including RCC $[29,30]$. For instance, the upregulation of the lncRNA MALAT1 is correlated with RCC progression and a poor prognosis [30].

Circular RNAs (circRNAs), a subclass of IncRNAs, are covalent single-chain closed-loop structures lacking terminal $5^{\prime}$ and $3^{\prime}$ ends [31]. Because of this extraordinary construction, circRNAs are resistant to degradation by exonucleases and are more stable than linear RNA. Thus, many studies have focused on the potential role of circRNAs as a promising disease biomarker. Further, studies in the past 10 years have highlighted the regulatory functions of circRNAs in both physiological and pathological settings [31]. Recently, the significance of circRNAs has been demonstrated in several cancers, including urinary system tumors such as bladder carcinoma [32] and prostate cancer [33]. Increasing evidence has witnessed that circRNAs play critical roles in RCC cell proliferation, apoptosis, migration, and invasion [34-50]. In this review, we summarize the circRNAs involved in RCC and their relevance to current clinical practice. A comprehensive understanding of circRNAs may provide valuable clues and useful information for future treatment options for RCC.

\section{Overview of circRNAs}

CircRNAs were originally dismissed as an artifact of transcription [51, 52]. Starting in 2010, assisted by highthroughput sequencing technology and bioinformatics, the number and types of identified circRNAs increased rapidly. In human tissues alone, in silico studies have predicted the existence of over 30,000 circRNAs, and subsequent studies on their biogenesis, characteristics, and functional mechanisms have been carried out [53-59].

In contrast to the canonical splicing of mRNAs, circRNAs are the result of a unique back-splicing process between the $5^{\prime}$ splice donor site and 3' splice acceptor site, which forms a continuous loop structure (Fig. 1). Different splicing processes result in three main types of circRNAs, including exonic circRNAs (ecircRNAs), exon-intron circRNAs (EIciRNAs), and circular intronic RNAs (ciRNAs). In addition, tRNA intronic circRNAs (tricRNAs) are generated by pre-tRNA splicing [60]. circRNA biogenesis can compete with linear pre-mRNA splicing, thereby affecting the alternative splicing of linear mRNAs and leading to altered gene expression. However, some studies have also shown that EIciRNAs and ciRNAs can enhance the transcription and splicing of their parental genes by interacting with RNA polymerase II (pol II) or the essential splicing factor U1 small nuclear ribonucleoprotein (snRNP) [61, 62]. Hundreds of circRNAs, encoded by the mitochondrial genome (mecciRNA) in both human and mouse cells, were recently identified [63]. The study found that some mecciRNAs can act as molecular chaperones to facilitate mitochondrial transport of proteins encoded by nuclear genes, which is essential for mitochondrial adaptation to physiological conditions. A study by $\mathrm{Su}$ et al. revealed that the novel mecciRNA SCAR has a critical role in nonalcoholic steatohepatitis (NASH) pathogenesis [64]. They showed that the inter-organelle metabolic axis could be disrupted with mitochondria-targeting nanoparticles (mito-NPs), which contained circRNA-expressing vectors and triphenylphosphonium (TPP)-decorated amphiphilic cationic peptides (TACP). mito-NPs can deliver circRNA SCAR specifically to the mitochondria, thereby alleviating metaflammation by reducing mROS output. This study highlighted a novel therapeutic strategy for immunometabolic and mitochondrial-related diseases.

The potential functions and biological activities of circRNAs have been extensively studied (Fig. 1). The beststudied function of circRNAs is to act as miRNAs sponges, thereby regulating downstream miRNA targets. For instance, circMTO1 targets miR-9, thereby inhibiting hepatocellular carcinoma proliferation and invasion by increasing p21, the target of oncogenic miR-9 [65]. Additionally, circRNAs can bind to numerous RNA-binding proteins (RBPs), acting as protein sponges or decoys to regulate protein functions [66]. A few circRNAs have been shown to serve as protein scaffolds, thereby promoting substrateenzyme binding and affecting the kinetics of the reaction $[67,68]$. Furthermore, under specific conditions, a subset of circRNAs can be translated into proteins [69], in a process that can be enhanced by the presence of $\mathrm{N}^{6}$-methyladenosine $\left(\mathrm{m}^{6} \mathrm{~A}\right)$ [70], thus adding another layer of complexity to circRNA regulation. 


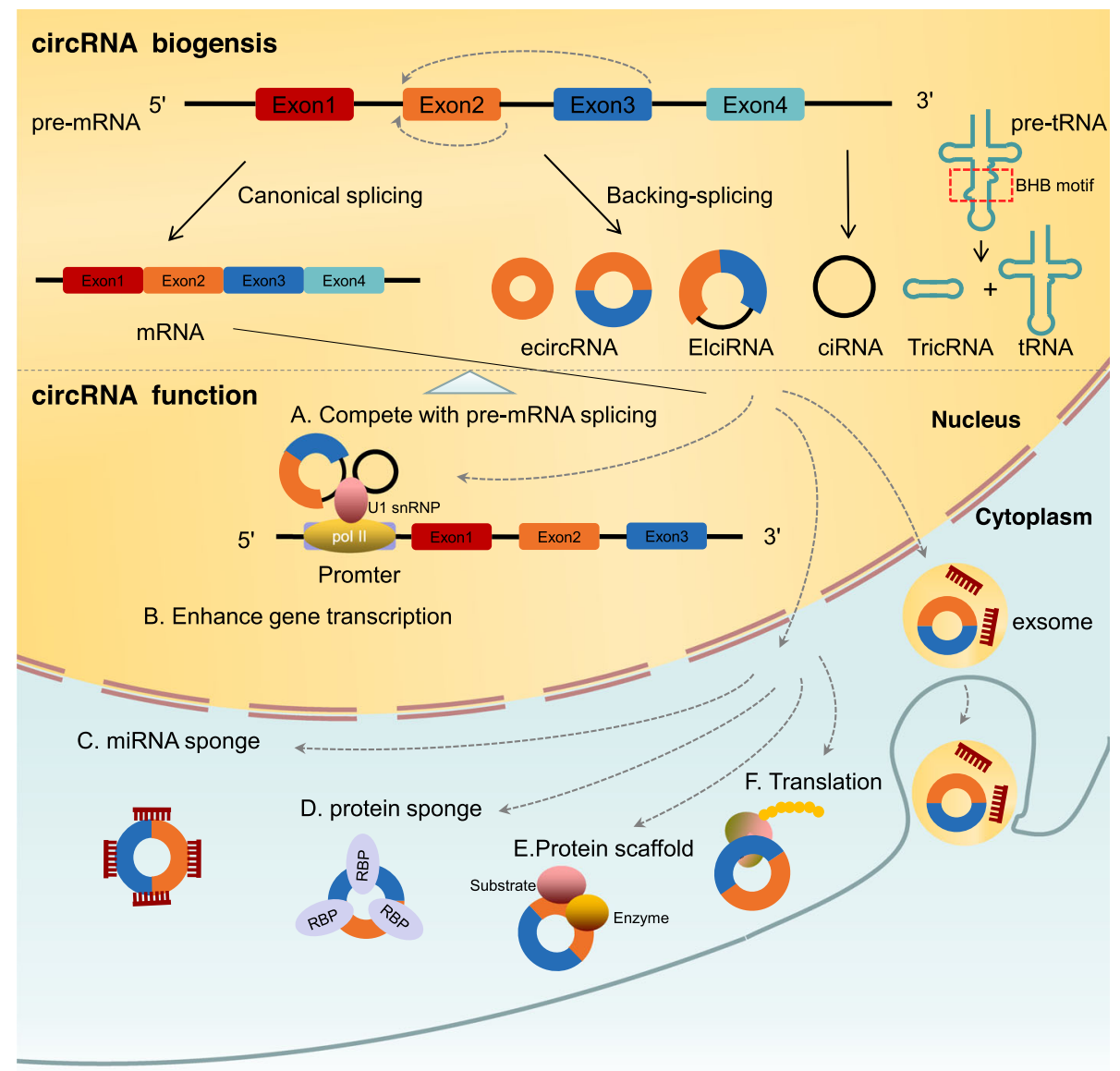

Fig. 1 Biogenesis and functions of circular RNAs (circRNAs). The biogenesis of circRNAs involves the noncanonical back-splicing process, which generates three types of circRNAs: ecircRNAs, ciRNAs, and ElciRNAs. TricRNAs are another type of circRNA that are generated via the splicing of pre-tRNA. CircRNA biogenesis competes with linear pre-mRNA splicing. CircRNAs enhance the transcription and splicing of their parental genes by interacting with RNA pol II or U1 small nuclear snRNP. CircRNAs act as miRNA sponges to regulate the expression of relevant target genes. CircRNAs bind to RBPs to mediate their actions. CircRNAs act as protein scaffolds to promote the binding of a substrate to an enzyme. CircRNAs can be translated into proteins

Because of their diverse functions, circRNAs not only regulate fundamental biological processes, but also play critical roles in a wide spectrum of human diseases including cancer [71-76]. Importantly, circRNAs are resistant to exoribonucleases and are therefore stable in the circulation, which makes them promising biomarkers for cancer diagnosis and prognosis.

\section{circRNA profiles in RCC}

The existence of circRNAs was first reported in viroids in 1976 [77]. In the past few years, research interest in circRNAs has significantly increased and many bioinformatics tools have been developed to accelerate these investigations [78-82]. Particularly, a few genome-wide transcriptional profiles of circRNAs in RCC have been reported. For instance, Ma et al. found that 542 circRNAs were aberrantly expressed in clear cell RCC (ccRCC) by using a circRNA microarray. Among these, 324 circRNAs were significantly downregulated, whereas 218 were upregulated in ccRCC tumors [83]. Using the Arraystar microarray to profile seven matched ccRCC samples, Franz et al. detected a total of 13,261 circRNAs, of which 78 circRNAs were upregulated and 91 were downregulated more than two-fold [84]. These high-throughput results strongly suggest a potential involvement of circRNAs in the pathogenesis of RCC, although their functions and underlying mechanisms are still being elucidated. Of note, different deregulated circRNAs were characterized in these studies, which might be associated with many factors, such as differences in clinical sample sources, sample processing methods, bioinformatics analyses, detection methods, and post-analytical validation.

\section{Dysregulation and molecular mechanisms of circRNAs in RCC}

Some circRNAs have been identified as key molecular regulators in the pathogenesis of RCC. The expression and functions of dysregulated circRNAs in RCC are 
Table 1 Overview of deregulated circRNAs in renal cell carcinoma (RCC)

\begin{tabular}{|c|c|c|c|c|c|}
\hline CircRNA & Expression Change & Function & Target microRNA & miRNA target genes/protein & Reference \\
\hline circPCNXL2 & up & $\begin{array}{l}\text { proliferation }(+) \\
\text { invasion }(+)\end{array}$ & miR-153 & ZEB2 & {$[34]$} \\
\hline circ-0039569 & up & $\begin{array}{l}\text { Proliferation }(+) \text {; migration }(+) \\
\text { invasion }(+)\end{array}$ & miR-34a-5p & CCL22 & {$[35]$} \\
\hline circ-ZNF609 & up & $\begin{array}{l}\text { proliferation }(+) \\
\text { invasion }(+)\end{array}$ & miR-138-5p & FOXP4 & {$[36]$} \\
\hline circNRIP1 & up & $\begin{array}{l}\text { proliferation }(+) \\
\text { migration }(+)\end{array}$ & miR-505 & AMPK and PI3K/AKT/mTOR & {$[37]$} \\
\hline circFNDC3B & up & cell viability (+); migration (+) & miR-99a & JAK1/STAT3 and MEK/ERK & {$[38]$} \\
\hline circ-001895 & up & $\begin{array}{l}\text { proliferation }(+) ; \\
\text { apoptosis }(-) ; \\
\text { migration }(+) ; \\
\text { invasion }(+)\end{array}$ & $\operatorname{miR}-296-5 p$ & SOX12 & {$[39]$} \\
\hline circ-EGLN3 & up & $\begin{array}{l}\text { proliferation }(+) ; \\
\text { apoptosis }(-) ; \\
\text { migration }(+) ; \\
\text { invasion }(+)\end{array}$ & miR-1299 & IRF7 & {$[40]$} \\
\hline circ-000926 & up & $\begin{array}{l}\text { proliferation }(+) ; \\
\text { migration }(+) ; \\
\text { invasion }(+) ; \\
\text { EMT }(+)\end{array}$ & miR-411 & $\mathrm{CDH} 2$ & [41] \\
\hline circ-ZNF652 & up & $\begin{array}{l}\text { proliferation }(+) ; \\
\text { apoptosis }(-) ; \\
\text { EMT }(+)\end{array}$ & miR-205 & Ras/Raf/MEK/ERK and JAK1/STAT3 & [42] \\
\hline circ-0035483 & up & $\begin{array}{l}\text { autophagy }(+) \text {; } \\
\text { tumor growth }(+) \text {; gemcitabine } \\
\text { resistance }(+)\end{array}$ & miR-335 & CCNB1 & [43] \\
\hline circ-ABCB10 & up & $\begin{array}{l}\text { proliferation }(+) ; \\
\text { apoptosis }(-)_{i} \\
\text { migration }(+)\end{array}$ & - & - & [44] \\
\hline circ-0072309 & down & $\begin{array}{l}\text { proliferation }(-) ; \\
\text { apoptosis }(+) ; \\
\text { migration }(-) \\
\text { invasion }(-)\end{array}$ & miR-100 & PI3K/AKT and mTOR & {$[45]$} \\
\hline circ-AKT3 & down & $\begin{array}{l}\text { migration }(-) \text {; } \\
\text { invasion }(-) \text {; } \\
\text { EMT }(-)\end{array}$ & miR-296-3p & E-cadherin & [46] \\
\hline CRAPGEF5 & down & $\begin{array}{l}\text { proliferation }(-) \\
\text { migration }(-) ; \\
\text { invasion }(-)\end{array}$ & $\operatorname{miR}-27 a-3 p$ & TXNIP & {$[47]$} \\
\hline circ-0001451 & down & $\begin{array}{l}\text { proliferation }(-) ; \\
\text { apoptosis }(+)\end{array}$ & - & - & {$[48]$} \\
\hline circHIAT1 & down & $\begin{array}{l}\text { migration }(-) \\
\text { invasion }(-)\end{array}$ & $\begin{array}{l}\text { miR-195-5p } \\
\text { miR-29a-3p } \\
\text { miR-29c-3p }\end{array}$ & $\mathrm{CDC} 42$ & {$[49]$} \\
\hline circATP2B1 & down & $\begin{array}{l}\text { migration (-); } \\
\text { invasion (-) }\end{array}$ & miR-204-3p & FN1 & {$[50]$} \\
\hline
\end{tabular}

listed in Table 1. These circRNAs participate in diverse processes of RCC pathogenesis, including proliferation, apoptosis, migration, invasion, epithelial-mesenchymal transition (EMT). Here, we discuss these circRNAs and their cellular functions and pathogenic mechanisms in RCC.

Most circRNAs promote the development and progression of RCC through circRNA-miRNA-mRNA interaction networks (Fig. 2). Some circRNAs act as tumor promoters in RCC cells. For instance, circZNF609 was highly expressed in multiple RCC cell lines compared with the normal renal epithelial cell line KiMA [36]. Further, circ-ZNF609 markedly promoted cell proliferation and invasion in RCC cells. Mechanistically, circ-ZNF609 can work as a miR-138-5p molecular sponge, which subsequently increases levels of FOXP4, 


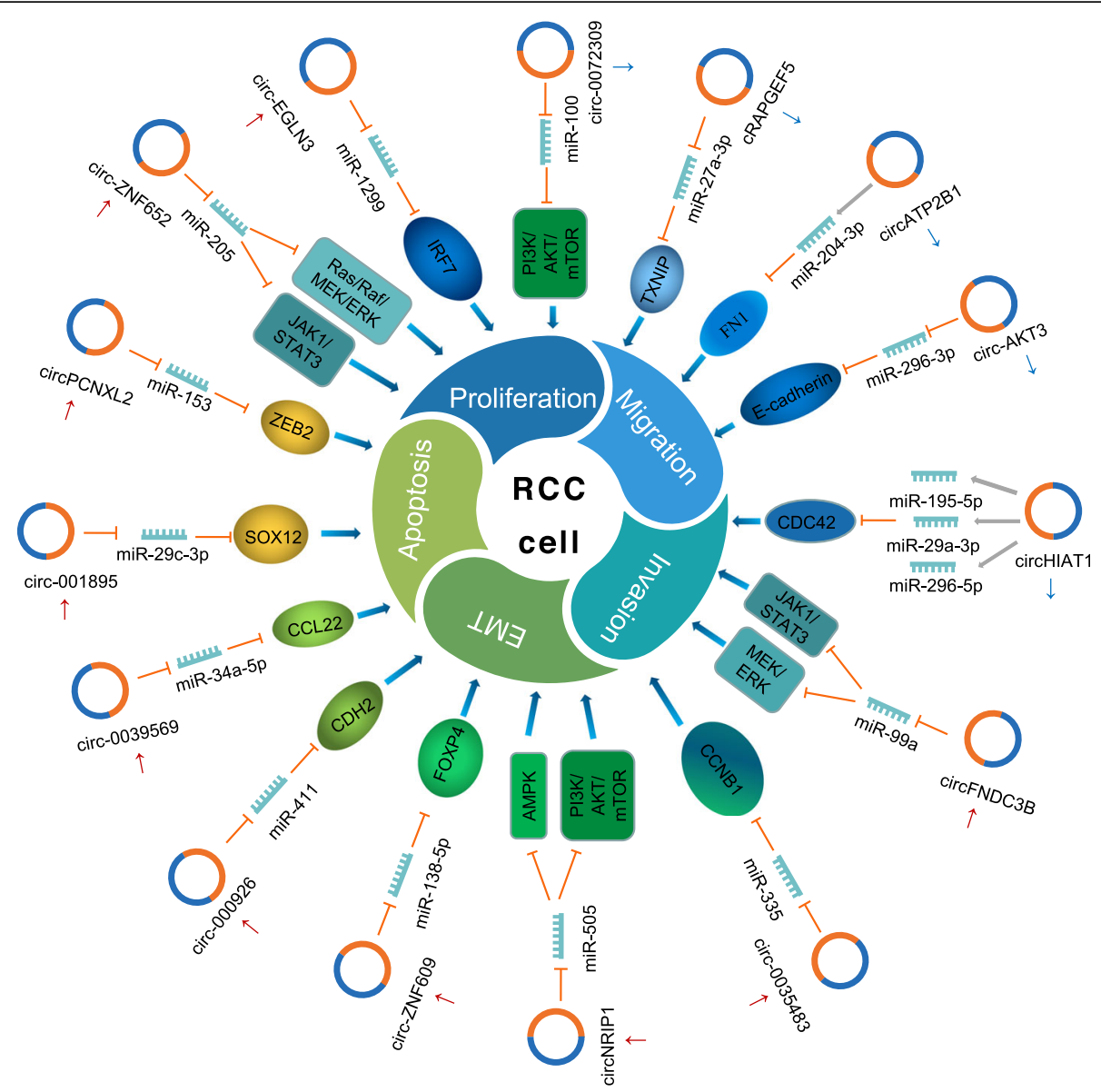

Fig. 2 Roles and regulatory pathways of RCC-related circRNAs. The schematic diagram shows the roles of circRNAs in RCC progression and the involvement of circRNAs in the miRNA-associated gene regulatory pathways

the known target of miR-138-5p. This study revealed a critical role of the circ-ZNF609/miR-138-5p/FOXP4 axis in RCC in vitro; however, its function in RCC progression in vivo awaits further investigation. Interestingly, circ-ZNF609 can be translated into a protein [85], in a process that may be regulated by $\mathrm{m}^{6} \mathrm{~A}$ modification [86]. Further investigation is needed to show whether this mode of circ-ZNF609 regulation also contributes to RCC. EMT plays a key role in tumor invasion and metastasis in multiple cancers, including RCC. Cadherin 2 (CDH2, also known as N-cadherin) is a marker of EMT and a major contributor to RCC aggressiveness that can be directly targeted by miR-411 [87-89]. A recent study reported that a novel circRNA (circ_000926) increased $\mathrm{CDH} 2$ expression by inhibiting miR-411 in RCC cells [41]. Moreover, silencing circ_000926 resulted in reduced cell proliferation, migration, and invasion in vitro, and markedly suppressed the growth and metastasis of RCC tumors in vivo. These results reveal a novel circ_000926/ miR-411/CDH2 regulatory axis in RCC tumorigenesis and metastasis. Other circRNAs have tumor suppressor roles in RCC cells [45-50]. Recently, the expression and function of cRAPGEF5 have implicated it as a novel tumor suppressor in RCC [47]. Reduced cRAPGEF5 expression in RCC was related to aggressive clinical characteristics. Functionally, silencing cRAPGEF5 promoted tumor growth and metastasis. RNA immunoprecipitation and in vitro biochemical assays showed that cRAPGEF5 functioned as an oncogenic miR-27a-3p sponge, which targets the tumor suppressor gene TXNIP. Like cRAPGEF5, circAKT3, which is transcribed from the AKT3 gene, was significantly reduced in RCC tumors and cell lines [46]. Overexpression of circAKT3 significantly inhibited ccRCC cell migration and invasion by sponging miR-296-3p, resulting in increased E-cadherin expression. Several other regulatory cascades comprising circRNA, miRNA, and mRNA have been reported in RCC including circPCNXL2/miR-153/ZEB2 [34], circ_0039569/miR-34a5p/CCL22 [35], hsa_circ_001895/miR-296-5p/SOX12 [39], and circEGLN/miR-1299/IRF7 [40].

Interestingly, some circRNAs can function as miRNA "reservoirs" to regulate RCC progression. The androgen receptor (AR) plays critical roles in the progression and metastasis of several hormone-regulated cancers 
including RCC. Wang et al. investigated the potential effects of functional interactions between AR and circRNAs in ccRCC [49]. Interestingly, circHIAT1 increased miR-195-5p/29a-3p/29c-3p activity by serving as a miRNA "reservoir", thereby inhibiting ARdependent migration and invasion of ccRCC cells. More recently, the novel circATP2B1 was also shown to regulate RCC progression by acting as a miRNA reservoir [50]. This study revealed that estrogen receptor $\beta$ (ER $\beta$ ) can promote ccRCC invasion by regulating the circATP2B1/miR-204-3p/FN1 signaling pathway. Overall, these studies illustrate that circRNAs have significant regulatory functions in RCC tumorigenesis.

In addition to miRNA regulation, recent evidence has demonstrated that some circRNAs regulate the proliferation and progression of RCC via cancer-associated signaling pathways (Table 1). For example, circNRIP1 plays an inhibitory role in RCC by regulating the adenosine monophosphate-activated protein kinase (AMPK) and PI3K/AKT/mTOR pathways [37]. Janus kinase/signal transducer and activator of transcription 3 (JAK/STAT3) and mitogen-activated protein/extracellular signalregulated kinase (MEK/ERK) signaling pathways are involved in RCC development. Chen et al. showed that circFNDC3B promoted cell viability and migration in RCC by activating the JAK1/STAT3 and MEK/ERK pathways [38]. Similarly, circZNF652 increased proliferation and EMT of RCC cells by regulating the Ras/Raf/ MEK/ERK and JAK1/STAT3 signaling pathways [42]. Although few circRNAs have been reported to play a role in RCC-associated signaling pathways to date, this list will undoubtedly continue to grow as more studies on the pathogenesis of RCC are carried out.

\section{circRNAs as diagnostic and prognostic biomarkers for RCC}

Screening and early diagnosis of RCC are critical for improving treatment efficacy and reducing the mortality of patients with RCC and have been identified as a research priority [90]. RCC can remain undetected for an extended period and only a small number of patients are diagnosed with RCC after presenting with classical symptoms, such as hematuria, flank pain, and a palpable abdominal mass [91]. Recently, with the widespread use of computed tomography (CT) and magnetic resonance imaging (MRI), incidental renal masses are increasingly being detected. However, there is an urgent need for specific biomarkers to allow for the early identification of postoperative RCC recurrence and metastasis. Notably, serum and urine analyses can be promising diagnostics because they are noninvasive and inexpensive.

circRNAs have great potential as a novel, attractive class of ncRNA biomarkers in liquid biopsy because they are resistant to RNase $\mathrm{R}$ digestion and remain stable in the circulation. circRNAs are abundant in body fluids, such as saliva, blood, and urine [92-95], and they can be detected using relatively inexpensive quantitative reverse transcriptase-polymerase chain reaction (qRT-PCR) assays [96]. Recent studies using clinical RCC samples demonstrated the abnormal expression, higher disease specificity, and clinical relevance of specific circRNAs, making them ideal candidates for RCC diagnosis. For instance, hsa_circ_0001451 was significantly decreased in ccRCC samples, and its level was linked to clinicopathological features and overall survival (OS), indicating the potential of this circRNA as a diagnostic marker for ccRCC. hsa_circ_0001451 had an area under the receiver operating characteristic curve (AUC-ROC) of 0.704 for discriminating ccRCC from normal controls, with sensitivity and specificity of 0.755 and 0.608 , respectively [48]. Franz et al. identified dysregulated circRNAs in ccRCC tissues by using a whole-genome microarray [84]. Three circRNAs (circEGLN3, circNOX4, and circRHOBTB3) were identified and clinically validated by qRT-PCR. Moreover, the AUC-ROC of circNOX4 and circRHOBTB3 in RCC tissues were 0.81 and 0.82 , suggesting that both could be used as potential diagnostic biomarkers. Importantly, circEGLN3 had AUC-ROC of 0.98 , indicating remarkably reliable diagnostic value. Further, the combined detection of circEGLN3 and linEGLN3 increased the AUC-ROC to 0.99, with 95\% sensitivity and $99 \%$ specificity. These results indicated that the diagnostic value of a combination of circRNAs is higher than that of individual circRNAs.

Additionally, some circRNAs could be used as prognostic biomarkers. Huang et al. showed that circABCB10 promoted RCC cell growth and suppressed apoptosis in vitro [44]. Moreover, circ-ABCB10 was markedly increased in RCC tissues and it was associated with pathologic grade and tumor-node-metastasis (TNM) stage. Therefore, circ-ABCB10 could be a prognostic factor for RCC. Another example is cRAPGEF5 [47]. In a study including 245 RCC cases, cRAPGEF5 downregulation was significantly associated with large tumor size, advanced TNM stage, and distant metastasis. Moreover, the level of cRAPGEF5 was correlated with aggressive tumor characteristics and poor OS and relapse-free survival (RFS) in patients, indicating that CRAPGEF5 could be used as a prognostic biomarker for RCC. circPRRC2A expression was increased and it was positively correlated with advanced TNM stage and lymph node metastasis in patients with RCC, indicating an oncogenic role. Furthermore, multivariate analyses indicated that circPRRC2A expression level was an independent risk factor for OS, together with RCC tumor size, pT stage, and Fuhrman grade. Kaplan-Meier survival curves showed that high circPRRC2A expression was associated with degreased with metastasis-free 
survival. These analyses also indicated that circPRRC2A may be a good prognostic biomarker for RCC [97].

The above examples demonstrated that some circRNAs could be promising biomarkers for the diagnosis and prognosis of RCC. However, these circRNAs are differentially expressed in tissues but are undetectable in plasma or serum. Can we develop liquid biopsy circRNAs biomarkers to distinguish healthy individuals from RCC patients and to correlate circRNA levels with tumor stages and metastasis risk? It is worth noting that there are still challenges hindering the clinical application of circRNAs as biomarkers. Monitoring biomarker research requires complex, large-scale prospective studies including the collection of continuous samples and the definition of time points and intervals, before circRNAs can be recommended for clinical use. Additionally, the normalization of circRNAs is another central point in liquid biopsies, which requires repeated tests to choose the optimal time and the cut-off value for their application to be compatible with the needs of patients. Despite these challenges, circRNAs are highly promising biomarker candidates, which can open more possibilities in RCC diagnosis and prognosis.

\section{circRNAs as potential therapeutic targets for RCC}

The emerging importance of circRNAs in the initiation and progression of RCC makes them an attractive therapeutic option [98-100]. For example, circHIAT1 and circATP2B1 could function as metastasis inhibitors to prevent AR- or ER $\beta$-induced ccRCC cell migration and invasion. Targeting these newly identified AR-circHIAT1- miR-195-5p/29a-3p/ 29c-3p/CDC42 and ER $\beta$-circATP2B1-mediated miR-2043p/FN1 signaling cascades can be a potential new route for development of therapies for metastatic ccRCC $[49,50]$.

Resistance to chemotherapeutic drugs is an intractable problem in RCC treatment. Gemcitabine is a novel type of drug that can selectively target tyrosine kinases [101]. Although gemcitabine has greatly improved RCC treatment, drug resistance consistently emerges after longterm application. Elucidating the molecular mechanism of gemcitabine resistance is important for RCC treatment. Recently, Yan et al. found that hsa-circ_0035483 was aberrantly expressed in RCC and enhanced gemcitabine resistance by regulating the hsa-miR-335/CCNB1 signaling pathway, thereby impairing chemotherapy effectiveness [43]. These results suggested that hsa_circ 0035483 could be a promising target for preventing gemcitabine resistance in RCC therapy. However, studies on circRNAs in RCC resistance to chemotherapy are still at a nascent stage. The connection between specific circRNAs and resistance to other classic drugs used in RCC treatment is still not clear. Undoubtedly, substantial efforts will be undertaken to reveal the function of circRNAs in RCC chemoresistance. Comprehensive evaluation of circRNA function in RCC will advance our knowledge of the mechanisms underlying $\mathrm{RCC}$ chemoresistance.

Several strategies for RCC treatment, which take into account the important functional roles of circRNAs as miRNA sponges, have been proposed. First, inhibiting or restoring circRNA function to regulate miRNAs and subsequently, their downstream targets, may be useful in RCC therapy. Thus far, several methods of inhibiting circRNA function have been developed [102], including siRNA, antisense oligonucleotide (ASO), CRISPR/Cas9mediated knockout, and disrupting the association of circRNAs with miRNAs or RBPs by saturating the conserved binding sites on circRNAs. In contrast, when circRNAs function as sponges for RBPs or miRNAs, restoring circRNA expression may inhibit abnormal proliferation or induce apoptosis. Thus, artificial circRNA sponges may be an effective approach to restore circRNA functions, providing a new strategy for drug development based on targeting miRNAs [76, 103, 104]. Furthermore, endogenous circRNAs are stable and have tissue- and cell-specific expression, suggesting that targeting circRNAs may be a future direction for gene therapy with potentially lower toxicity than that of synthetic molecules, such as siRNAs and synthetic drugs.

Exploring the unique properties of circRNAs is a novel frontier in RCC treatment. Engineered circRNAs could be an accurate and effective method for delivering therapeutic agents. For instance, high-quality protein translation was achieved by using an artificial circRNA and protein half-life was extended threefold [105]. In addition, the Tornado expression system has been developed to efficiently increase circRNA expression in cells [106]. At present, although such circRNAs have not been found in RCC, further research may identify circRNA with promising application potential for RCC therapy. Overall, research on circRNAs as potential therapeutic targets or useful tools is a rapidly developing field in oncology.

\section{Challenges and future perspectives}

Currently, interest in circRNAs is growing and their role in RCC is becoming better understood. However, the clinical application of circRNAs in RCC remains largely unexplored and further research is needed prior to incorporating circRNAs into clinical practice.

Only a few circRNAs have defined biological functions and molecular mechanisms in RCC and the exact mechanisms of circRNA circularization, degradation, and cellular localization in RCC need further investigation. Due to potential alternative splicing, the internal structure of circRNAs has remained ill-defined, which has impeded research on circRNA function. Notably, Feng et al. developed a new algorithm, CircSplice, which could 
identify internal alternative splicing in circRNAs and compare differential circRNA splicing events between different conditions [107]. The application of this algorithm in ccRCC characterized patterns of cancer-specific circRNA alternative splicing (circ-AS), providing a useful resource to explore the functional and regulatory roles of circRNAs in RCC. At present, most studies on circRNAs are focused on their function as miRNA sponges that regulate the expression of the targeted genes. In addition to this regulatory mechanism, other mechanisms may also be involved in the regulation of circRNA function. It is unclear whether circRNAs can be regulated through different molecular regulatory mechanisms at the same time. Further, many studies have confirmed that the tumor microenvironment can impact tumor initiation and progression; however, the effects of circRNAs on the RCC microenvironment have remained elusive until recently $[108,109]$. Therefore, elucidating the role of circRNAs in the tumor microenvironment will further clarify the underlying mechanisms of RCC initiation and progression, thereby identifying possible targets for therapeutic intervention.

Although several circRNAs can be potential tumor biomarkers, current research investigating circRNAs as RCC biomarkers is limited. Currently, most circRNA biomarkers may not be suitable for clinical application since they are lacking in sensitivity or specificity. Importantly, more clinical studies on circRNAs as biomarkers should be carried out and will require standardized techniques and bioinformatics methods to reliably detect circRNAs.

Ultimately, more research is needed on how to efficiently deliver circRNAs to recipient cells to regulate cancer progression without immunologic rejection and with sustained long-term effects. Exosomes may greatly expand the applications of circRNAs. Exosomes are small membrane vesicles released by most cell types including tumor cells, which can act as natural intercellular communication mediators by transporting signaling molecules, including circRNAs, to target tissues. Recently, exosomes have been confirmed to contain stable and abundant circRNAs [110]. Intriguingly, serum exosomal circRNAs can distinguish patients with colon cancer from healthy controls [110]. However, the biological functions of exosomal circRNAs in RCC progression need further exploration.

\section{Conclusions}

In this review, we discussed and summarized the recent discoveries and research progress on RCC-associated circRNAs, and further emphasized their potential clinical applications, which provides a new direction for developing RCC diagnostics and therapies. However, c circRNAs contribute to RCC initiation, and progression is not fully understood. It is highly anticipated that
circRNA-based diagnostic and therapeutic interventions for RCC will emerge in the near future.

\begin{abstract}
Abbreviations
RCC: Renal cell carcinoma; circRNAs: Circular RNAs; ncRNAs: Non-coding RNAs; miRNAs: MicroRNAs; IncRNAs: Long-noncoding RNAs; tsRNAs: Transfer RNA [tRNA]-derived small RNAs; piRNAs: PIWl-interacting RNAs;

ecircRNAs: Exonic circRNAs; ElciRNAs: Exon-intron circRNAs; ciRNAs: Intronic circRNAs; tricRNAs: CircRNAs formed by the splicing of pre-tRNA; Pol II: RNA polymerase II; snRNP: Small nuclear ribonucleoprotein;

mecciRNA: Mitochondrial circRNA; NASH: Nonalcoholic steatohepatitis; mitoNP: Mitochondria-targeting nanoparticles; RBPs: RNA-binding proteins; ceRNA: Competing endogenous RNA; ccRCC: Renal clear cell carcinoma; EMT: Epithelial-mesenchymal transition; TNM: Tumor-node-metastasis; AR: Androgen receptor; ERß: Estrogen receptor beta; qRT-PCR: Quantitative reverse transcriptase-polymerase chain reaction; OS: Overall survival; RFS: Relapse-free survival; ROC: Receiver operating characteristic; GEO: Gene Expression Omnibus; ASOs: Anti-sense oligonucleotides; CRISPR/

Cas9: Clustered regularly interspaced short palindromic repeats-associated nuclease Cas9; circ-AS: CircRNA alternative splicing
\end{abstract}

\section{Acknowledgments}

Not applicable.

\section{Authors' contributions}

WL, PW and XF conceived the structure of the manuscript; YW drafted the initial manuscript; $W L$ and $Y Z$ revised the manuscript. All authors have contributed to the preparation of this manuscript. Both authors read and approved the final manuscript.

\section{Funding}

This work was supported by grants from the National Key R\&D Program of China (2018YFC2000400), and the National Natural Science Foundation of China (31470776, 81670651, 81970573, and 81772270).

Availability of data and materials

Not applicable.

\section{Ethics approval and consent to participate}

Not applicable.

\section{Consent for publication}

Not applicable.

\section{Competing interests}

The authors declare that they have no competing interests.

Received: 15 April 2020 Accepted: 2 October 2020

Published online: 14 October 2020

References

1. Motzer RJ, Bander NH, Nanus DM. Renal-cell carcinoma. N Engl J Med. 1996; 335:865-75

2. Siegel RL, Miller KD, Jemal A. Cancer statistics, 2020. CA Cancer Clin. 2020;70: 7-30.

3. Znaor A, Lortet-Tieulent J, Laversanne M, Jemal A, Bray F. International variations and trends in renal cell carcinoma incidence and mortality. Eur Urol. 2015;67:519-30.

4. Welch HG, Skinner JS, Schroeck FR, Zhou W, Black WC. Regional variation of computed tomographic imaging in the United States and the risk of nephrectomy. JAMA Intern Med. 2018;178:221-7.

5. Lieder A, Guenzel T, Lebentrau S, Schneider C, Franzen A. Diagnostic relevance of metastatic renal cell carcinoma in the head and neck: an evaluation of 22 cases in 671 patients. Int Braz J rol. 2017;43:202-8.

6. Lara PN Jr, Evans CP. Cytoreductive nephrectomy in metastatic renal cell Cancer: not all that It's cut out to be. JAMA Oncol. 2019:5:171-2.

7. Pal SK, Agarwal N. Kidney cancer: finding a niche for girentuximab in metastatic renal cell carcinoma. Nat Rev Urol. 2016;13:442-3.

8. Benhaim R, Oussoultzoglou E, Saeedi Y, Mouracade P, Bachellier P, Lang H Pancreatic metastasis from clear cell renal cell carcinoma: outcome of an aggressive approach. Urology. 2015;85:135-40. 
9. Petejova N, Martinek A. Renal cell carcinoma: review of etiology, pathophysiology and risk factors. Biomed Pap Med Fac Univ Palacky Olomouc Czech Repub. 2016;160:183-94.

10. Crispen $\mathrm{PL}$, Breau RH, Allmer C, Lohse CM, Cheville JC, Leibovich BC, et al. Lymph node dissection at the time of radical nephrectomy for high-risk clear cell renal cell carcinoma: indications and recommendations for surgical templates. Eur Urol. 2011;59:18-23.

11. Barata PC, Rini BI. Treatment of renal cell carcinoma: current status and future directions. CA Cancer J Clin. 2017:67:507-24.

12. Perazella MA, Dreicer $\mathrm{R}$, Rosner $\mathrm{MH}$. Renal cell carcinoma for the nephrologist. Kidney Int. 2018;94:471-83.

13. Capitanio U, Bensalah K, Bex A, Boorjian SA, Bray F, Coleman J, et al. Epidemiology of renal cell carcinoma. Eur Urol. 2019;75:74-84.

14. Cancer Genome Atlas Research Network. Comprehensive molecular characterization of clear cell renal cell carcinoma. Nature. 2013;499:43-9.

15. Carril-Ajuria L, Santos M, Roldán-Romero JM, Rodriguez-Antona C, de Velasco G. Prognostic and Predictive Value of PBRM1 in Clear Cell Renal Cell Carcinoma. Cancers (Basel). 2019;12:16.

16. Li L, Miao W, Huang M, Williams P, Wang Y. Integrated genomic and proteomic analyses reveal novel mechanisms of the methyltransferase SETD2 in renal cell carcinoma development. Mol Cell Proteomics. 2019;18: 437-47.

17. Di Cristofano C, Minervini A, Menicagli M, Salinitri G, Bertacca G, Pefanis G, et al. Nuclear expression of hypoxia-inducible factor-1alpha in clear cell renal cell carcinoma is involved in tumor progression. Am J Surg Pathol. 2007;31:1875-81.

18. Kim J, Ulu A, Wan D, Yang J, Hammock BD, Weiss RH. Addition of DHA synergistically enhances the efficacy of Regorafenib for kidney Cancer therapy. Mol Cancer Ther. 2016;15:890-8.

19. Shang D, Liu Y, Ito N, Kamoto T, Ogawa O. Defective JakStat activation in renal cell carcinoma is associated with interferon-alpha resistance. Cancer Sci. 2007:98:1259-64

20. Xu Q, Krause M, Samoylenko A, Vainio S. Wnt Signaling in Renal Cell Carcinoma. Cancers (Basel). 2016:8:57.

21. Zhai $W$, Sun $Y$, Jiang $M$, Wang $M$, Gasiewicz TA, Zheng J, et al. Differential regulation of LnCRNA-SARCC suppresses VHL-mutant RCC cell proliferation yet promotes $\mathrm{VHL}$-normal RCC cell proliferation via modulating androgen receptor/HIF-2a/C-MYC axis under hypoxia. Oncogene. 2016;35:4866-80.

22. Elfiky AA, Aziz SA, Conrad PJ, Siddiqui S, Hackl W, Maira M, et al. Characterization and targeting of phosphatidylinositol-3 kinase (PI3K) and mammalian target of rapamycin (mTOR) in renal cell cancer. J Transl Med. 2011:9:133.

23. Li M, Wang $Y$, Song $Y, B u R$, Yin $B$, Fei $X$, et al. Expression profiling and clinicopathological significance of DNA methyltransferase 1, 3A and 3B in sporadic human renal cell carcinoma. Int J Clin Exp Pathol. 2014;7:7597-609.

24. Mosashvilli D, Kahl P, Mertens C, Holzapfel S, Rogenhofer S, Hauser S, et al. Global histone acetylation levels: prognostic relevance in patients with renal cell carcinoma. Cancer Sci. 2010;101:2664-9.

25. Li M, Wang $Y$, Song $Y, B u R$, Yin B, Fei $X$, et al. MicroRNAs in renal cell carcinoma: a systematic review of clinical implications (review). Oncol Rep. 2015;33:1571-8.

26. Slack FJ, Chinnaiyan AM. The role of non-coding RNAs in oncology. Cell. 2019:179:1033-55

27. Dang K, Myers KA. The role of hypoxia-induced miR-210 in cancer progression. Int J Mol Sci. 2015;16:6353-72.

28. Grange C, Brossa A, Bussolati B. Extracellular vesicles and carried miRNAs in the progression of renal cell carcinoma. Int J Mol Sci. 2019;20:1832.

29. Zhai W, Zhu R, Ma J, Gong D, Zhang H, Zhang J, et al. A positive feedforward loop between LnCRNA-URRCC and EGFL7/P-AKT/FOXO3 signaling promotes proliferation and metastasis of clear cell renal cell carcinoma. Mol Cancer. 2019;18:81

30. Li Z, Ma Z, Xu X. Long non-coding RNA MALAT1 correlates with cell viability and mobility by targeting miR-22-3p in renal cell carcinoma via the PI3KJAkt pathway. Oncol Rep. 2019;41:1113-21.

31. Patop IL, Wüst S, Kadener S. Past, present, and future of circRNAs. EMBO J. 2019:38:e100836

32. Su H, Tao T, Yang Z, Kang X, Zhang X, Kang D, et al. Circular RNA cTFRC acts as the sponge of MicroRNA-107 to promote bladder carcinoma progression. Mol Cancer. 2019;18:27.

33. Hua JT, Chen S, He HH. Landscape of noncoding RNA in prostate Cancer. Trends Genet. 2019;35:840-51.
34. Zhou B, Zheng P, Li Z, Li H, Wang X, Shi Z, et al. CircPCNXL2 sponges miR153 to promote the proliferation and invasion of renal cancer cells through upregulating ZEB2. Cell Cycle. 2018;17:2644-54.

35. Jin C, Shi L, Li Z, Liu W, Zhao B, Qiu Y, et al. Circ_0039569 promotes renal cell carcinoma growth and metastasis by regulating miR-34a-5p/CCL22. Am J Transl Res. 2019;11:4935-45.

36. Xiong $Y$, Zhang J, Song C. CircRNA ZNF609 functions as a competitive endogenous RNA to regulate FOXP4 expression by sponging miR-138-5p in renal carcinoma. J Cell Physiol. 2019;234:10646-54.

37. Dong Z, Liu Y, Wang Q, Wang H, Ji J, Huang T, et al. The circular RNA-NRIP1 plays oncogenic roles by targeting microRNA-505 in the renal carcinoma cell lines. J Cell Biochem. 2020;121:2236-46.

38. Chen T, Yu Q, Shao S, Guo L. Circular RNA circFNDC3B protects renal carcinoma by miR-99a downregulation. J Cell Physiol. 2020;235:4399-406.

39. Chen Z, Xiao K, Chen S, Huang Z, Ye Y, Chen T. Circular RNA hsa_circ_001895 serves as a sponge of microRNA-296-5p to promote clear cell renal cell carcinoma progression by regulating SOX12. Cancer Sci. 2020;111:713-26.

40. Lin L, Cai J. Circular RNA circ-EGLN3 promotes renal cell carcinoma proliferation and aggressiveness via miR-1299-mediated IRF7 activation. J Cell Biochem. 2020. https://doi.org/10.1002/jcb.29620.

41. Zhang D, Yang XJ, Luo QD, Fu DL, Li ZL, Zhang P, et al. Down-regulation of circular RNA_000926 attenuates renal cell carcinoma progression through miRNA-411-dependent CDH2 inhibition. Am J Pathol. 2019;189:2469-86.

42. Zhang $L$, Guo Y. Silencing circular RNA-ZNF652 represses proliferation and EMT process of renal carcinoma cells via raising miR-205. Artif Cells Nanomed Biotechnol. 2020:48:648-55.

43. Yan L, Liu G, Cao H, Zhang H, Shao F. Hsa_circ_0035483 sponges hsa-miR335 to promote the gemcitabine-resistance of human renal cancer cells by autophagy regulation. Biochem Biophys Res Commun. 2019;519:172-8.

44. Huang Y, Zhang Y, Jia L, Liu C, Xu F. Circular RNA ABCB10 promotes tumor progression and correlates with pejorative prognosis in clear cell renal cell carcinoma. Int J Biol Markers. 2019;34:176-83.

45. Chen T, Shao S, Li W, Liu Y, Cao Y. The circular RNA hsa-circ-0072309 plays anti-tumour roles by sponging miR-100 through the deactivation of PI3K AKT and mTOR pathways in the renal carcinoma cell lines. Artif Cells Nanomed Biotechnol. 2019:47:3638-48.

46. Xue D, Wang H, Chen Y, Shen D, Lu J, Wang M, et al. Circ-AKT3 inhibits clear cell renal cell carcinoma metastasis via altering miR-296-3p/E-cadherin signals. Mol Cancer. 2019;18:151.

47. Chen Q, Liu T, Bao Y, Zhao T, Wang J, Wang H, et al. CircRNA CRAPGEF5 inhibits the growth and metastasis of renal cell carcinoma via the miR-27a3p/TXNIP pathway. Cancer Lett. 2020;469:68-77.

48. Wang G, Xue W, Jian W, Liu P, Wang Z, Wang C, et al. The effect of Hsa circ 0001451 in clear cell renal cell carcinoma cells and its relationship with clinicopathological features. J Cancer. 2018;9:3269-77.

49. Wang K, Sun Y, Tao W, Fei $X$, Chang C. Androgen receptor (AR) promotes clear cell renal cell carcinoma (ccRCC) migration and invasion via altering the circHIAT1/miR-195-5p/29a-3p/29c-3p/CDC42 signals. Cancer Lett. 2017; 394:1-12.

50. Han Z, Zhang Y, Sun Y, Chen J, Chang C, Wang X, et al. ERß-mediated alteration of circATP2B1 and miR-204-3p signaling promotes invasion of clear cell renal cell carcinoma. Cancer Res. 2018;78:2550-63.

51. Tan WL, Lim BT, Anene-Nzelu CG, Ackers-Johnson M, Dashi A, See K, et al. A landscape of circular RNA expression in the human heart. Cardiovasc Res. 2017;113:298-309.

52. Capel B, Swain A, Nicolis S, Hacker A, Walter M, Koopman P, et al. Circular transcripts of the testis-determining gene Sry in adult mouse testis. Cell. 1993;73:1019-30.

53. Hansen $\mathrm{TB}$, Jensen $\mathrm{TI}$, Clausen BH, Bramsen JB, Finsen B, Damgaard CK, et al. Natural RNA circles function as efficient microRNA sponges. Nature. 2013; 495:384-8.

54. Kramer MC, Liang D, Tatomer DC, Gold B, March ZM, Cherry S, et al. Combinatorial control of Drosophila circular RNA expression by intronic repeats, hnRNPs, and SR proteins. Genes Dev. 2015:29:2168-82.

55. Memczak S, Jens M, Elefsinioti A, Torti F, Krueger J, Rybak A, et al. Circular RNAs are a large class of animal RNAs with regulatory potency. Nature. 2013:495:333-8

56. Pan T, Sun X, Liu Y, Li H, Deng G, Lin H, et al. Heat stress alters genomewide profiles of circular RNAs in Arabidopsis. Plant Mol Biol. 2018;96:217-29.

57. Wang PL, Bao Y, Yee MC, Barrett SP, Hogan GJ, Olsen MN, et al. Circular RNA is expressed across the eukaryotic tree of life. PLoS One. 2014;9:e90859. 
58. Li $X$, Yang $L$, Chen LL. The biogenesis, functions, and challenges of circular RNAs. Mol Cell. 2018;71:428-42

59. Szabo L, Salzman J. Detecting circular RNAs: bioinformatic and experimental challenges. Nat Rev Genet. 2016;17:679-92.

60. Huang G, Li S, Yang N, Zou Y, Zheng D, Xiao T. Recent progress in circular RNAs in human cancers. Cancer Lett. 2017;404:8-18.

61. Zhang Y, Zhang XO, Chen T, Wang J, Gao Y, Shang R, et al. Circular intronic long noncoding RNAs. Mol Cell. 2013;51:792-806.

62. Li Z, Huang C, Bao C, Chen L, Lin M, Wang X, et al. Exon-intron circular RNAs regulate transcription in the nucleus. Nat Struct Mol Biol. 2015;22:256-64.

63. Liu X, Wang X, Li J, Hu S, Deng Y, Yin H, et al. Identification of mecciRNAs and their roles in the mitochondrial entry of proteins. Sci China Life Sci. 2020;63:1429-49.

64. Zhao Q, Liu J, Deng H, Ma R, Liao JY, Liang H, et al. Targeting MitochondriaLocated circRNA SCAR AlleviatesNASH via Reducing mROS Output. Cell. 2020;183(1):76-93.e22

65. Han D, Li J, Wang H, Su X, Hou J, Gu Y, et al. Circular RNA circMTO1 acts as the sponge of microRNA-9 to suppress hepatocellular carcinoma progression. Hepatology. 2017;66:1151-64.

66. Dudekula DB, Panda AC, Grammatikakis I, De S, Abdelmohsen K, Gorospe M. Circlnteractome: a web tool for exploring circular RNAs and their interacting proteins and microRNAs. RNA Biol. 2016;13:34-42.

67. Zeng Y, Du WW, Wu Y, Yang Z, Awan FM, Li X, et al. A circular RNA binds to and activates AKT phosphorylation and nuclear localization reducing apoptosis and enhancing cardiac repair. Theranostics. 2017;7:3842-55.

68. Du WW, Fang L, Yang W, Wu N, Awan FM, Yang Z, et al. Induction of tumor apoptosis through a circular RNA enhancing Foxo3 activity. Cell Death Differ. 2017;24:357-70.

69. Kristensen LS, Andersen MS, Stagsted LW, Ebbesen KK, Hansen TB, Kjems J. The biogenesis, biology and characterization of circular RNAs. Nat Rev Genet. 2019;20:675-91

70. Yang $Y$, Fan $X$, Mao $M$, Song $X$, Wu $P$, Zhang $Y$, et al. Extensive translation of circular RNAs driven by N6-methyladenosine. Cell Res. 2017;27:626-41.

71. Song M, Xia L, Sun M, Yang C, Wang F. Circular RNA in liver: health and diseases. Adv Exp Med Biol. 2018;1087:245-57.

72. Altesha MA, Ni T, Khan A, Liu K, Zheng X. Circular RNA in cardiovascular disease. J Cell Physiol. 2019;234:5588-600.

73. Arnaiz E, Sole C, Manterola L, Iparraguirre L, Otaegui D, Lawrie CH. CircRNAs and cancer: biomarkers and master regulators. Semin Cancer Biol. 2019;58: 90-9.

74. Fang $Y$, Wang $X$, Li W, Han J, Jin J, Su F, et al. Screening of circular RNAs and validation of circANKRD36 associated with inflammation in patients with type 2 diabetes mellitus. Int J Mol Med. 2018;42:1865-74.

75. Hanan M, Soreq H, Kadener S. CircRNAs in the brain. RNA Biol. 2017;14: 1028-34.

76. Kristensen LS, Hansen TB, Venø MT, Kjems J. Circular RNAs in cancer: opportunities and challenges in the field. Oncogene. 2018:37:555-65..

77. Sanger HL, Klotz G, Riesner D, Gross HJ, Kleinschmidt AK. Viroids are singlestranded covalently closed circular RNA molecules existing as highly basepaired rod-like structures. Proc Natl Acad Sci U S A. 1976;73:3852-6.

78. Maass PG, Glažar P, Memczak S, Dittmar G, Hollfinger I, Schreyer L, et al. A map of human circular RNAs in clinically relevant tissues. J Mol Med (Berl). 2017:95:1179-89.

79. Liang J, Wu X, Sun S, Chen P, Liang X, Wang J, Ruan J, et al. Circular RNA expression profile analysis of severe acne by RNA-Seq and bioinformatics. J Eur Acad Dermatol Venereol. 2018;32:1986-92.

80. López-Jiménez E, Rojas AM, Andrés-León E. RNA sequencing and prediction tools for circular RNAs analysis. Adv Exp Med Biol. 2018;1087:17-33.

81. Vromman M, Vandesompele J, Volders PJ. Closing the circle: current state and perspectives of circular RNA databases. Brief Bioinform. 2020; 21(bbz175):1-10.

82. Feng J, Xiang Y, Xia S, Liu H, Wang J, Ozguc FM, Lei L, Kong R, Diao L, He C, Han L. CircView: a visualization and exploration tool for circular RNAs. Brief Bioinform. 2018;19:1310-6.

83. Ma C, Qin J, Zhang J, Wang X, Wu D, Li X. Construction and analysis of circular RNA molecular regulatory networks in clear cell renal cell carcinoma. Mol Med Rep. 2020;21:141-50.

84. Franz A, Ralla B, Weickmann S, Jung M, Rochow H, Stephan C, et al. Circular RNAs in Clear Cell Renal Cell Carcinoma: Their Microarray-Based Identification, Analytical Validation, and Potential Use in a Clinico-Genomic Model to Improve Prognostic Accuracy. Cancers (Basel). 2019;11:1473.
85. Legnini I, Di Timoteo G, Rossi F, Morlando M, Briganti F, Sthandier O, et al. Circ-ZNF609 Is a Circular RNA that Can Be Translated and Functions in Myogenesis. Mol Cell. 2017;66:22-37 e9.

86. Di Timoteo G, Dattilo D, Centrón-Broco A, Colantoni A, Guarnacci M, Rossi F, et al. Modulation of circRNA metabolism by m6A modification. Cell Rep. 2020;31:107641.

87. Alimperti $\mathrm{S}$, Andreadis ST. $\mathrm{CDH} 2$ and $\mathrm{CDH} 11$ act as regulators of stem cell fate decisions. Stem Cell Res. 2015;14:270-82.

88. Chen D, Zhu M, Su H, Chen J, Xu X, Cao C. LINC00961 restrains cancer progression via modulating epithelial-mesenchymal transition in renal cell carcinoma. J Cell Physiol. 2019;234:7257-65.

89. Han X, Piao L, Yuan X, Wang L, Liu Z, He X. Knockdown of NSD2 suppresses renal cell carcinoma metastasis by inhibiting epithelial-Mesenchymal transition. Int J Med Sci. 2019;16:1404-11.

90. Porta C, Gore ME, Rini BI, Escudier B, Hariharan S, Charles LP, et al. Longterm safety of Sunitinib in metastatic renal cell carcinoma. Eur Urol. 2016;69: 345-51.

91. Rossi SH, Klatte T, Usher-Smith J, Stewart GD. Epidemiology and screening for renal cancer. World J Urol. 2018;36:1341-53.

92. Jafari GF. Circular RNA in saliva. Adv Exp Med Biol. 2018;1087:131-9.

93. Fanale D, Taverna S, Russo A, Bazan V. Circular RNA in Exosomes. Adv Exp Med Biol. 2018;1087:109-17.

94. Vea A, Llorente-Cortes V, de Gonzalo-Calvo D. Circular RNAs in blood. Adv Exp Med Biol. 2018;1087:119-30.

95. Kölling M, Haddad G, Wegmann U, Kistler A, Bosakova A, Seeger H, et al. Circular RNAs in urine of kidney transplant patients with acute T cellmediated allograft rejection. Clin Chem. 2019;65:1287-94.

96. Vo JN, Cieslik M, Zhang Y, Shukla S, Xiao L, Zhang Y, et al. The Landscape of Circular RNA in Cancer. Cell. 2019;176:869-81 e13.

97. Li W, Yang FQ, Sun CM, Huang JH, Zhang HM, Li X, et al. CircPRRC2A promotes angiogenesis and metastasis through epithelial-mesenchymal transition and upregulates TRPM3 in renal cell carcinoma. Theranostics. 2020;10:4395-409.

98. Jiang $W D$, Ye $Z \mathbf{H}$. Integrated analysis of a competing endogenous RNA network in renal cell carcinoma using bioinformatics tools. Biosci Rep. 2019; 39:BSR20190996

99. Liu J, Liu T, Wang $X$, He A. Circles reshaping the RNA world: from waste to treasure. Mol Cancer. 2017;16:58.

100. Yang Z, Xie L, Han L, Qu X, Yang Y, Zhang Y, et al. Circular RNAs: regulators of Cancer-related signaling pathways and potential diagnostic biomarkers for human cancers. Theranostics. 2017;7:3106-17.

101. Yang D, Tang Y, Fu H, Xu J, Hu Z, Zhang Y, Cai Q. Integrin $\beta 1$ promotes gemcitabine resistance in pancreatic cancer through Cdc42 activation of PI3K p110ß signaling. Biochem Biophys Res Commun. 2018;505:215-21.

102. Zhang M, Xin Y. Circular RNAs: a new frontier for cancer diagnosis and therapy. J Hematol Oncol. 2018;11:21.

103. Holdt LM, Kohlmaier A, Teupser D. Circular RNAs as therapeutic agents and targets. Front Physiol. 2018;9:1262.

104. Rossbach O. Artificial circular RNA sponges targeting MicroRNAs as a novel tool in molecular biology. Mol Ther Nucleic Acids. 2019;17:452-4.

105. Wesselhoeft RA, Kowalski PS, Anderson DG. Engineering circular RNA for potent and stable translation in eukaryotic cells. Nat Commun. 2018;9:2629.

106. Litke $J$, Jaffrey SR. Highly efficient expression of circular RNA aptamers in cells using autocatalytic transcripts. Nat Biotechnol. 2019;37:667-75.

107. Feng J, Chen K, Dong $X, X u X$, Jin Y, Zhang X, et al. Genome-wide identification of cancer-specific alternative splicing in circRNA. Mol Cancer. 2019;18:35.

108. Lee $K$, Hwang H, Nam KT. Immune response and the tumor microenvironment: how they communicate to regulate gastric cancer. Gut Liver. 2014;8:131-9.

109. Ma Z, Shuai Y, Gao X, Wen X, Ji J. Circular RNAs in the tumour microenvironment. Mol Cancer. 2020;19:8.

110. Li Y, Zheng Q, Bao C, Li S, Guo W, Zhao J, et al. Circular RNA is enriched and stable in exosomes: a promising biomarker for cancer diagnosis. Cell Res. 2015;25:981-4.

\section{Publisher's Note}

Springer Nature remains neutral with regard to jurisdictional claims in published maps and institutional affiliations. 